\title{
ANESTESIA POR INFUSÃO CONTÍNUA DE PROPOFOL EM CÃES PRÉ-MEDICADOS COM ACEPROMAZINA E FENTANIL
}

\section{ANESTHESIA BY CONTINUOUS INFUSION OF PROPOFOL IN DOGS PREMEDICATED WITH ACEPROMAZINE AND FENTANYL}

\author{
Jefferson da Silva Pires ${ }^{1}$, Rui Afonso Viera Campello², Renato Xavier Faria ${ }^{3}$,
}

Alonso Gabriel Pereira Guedes ${ }^{4}$

RESUMO

\begin{abstract}
O propofol (2,6 diisopropilfenol) é um agente hipnótico de ultra curta duração que produz sedação e hipnose similar aos barbitúricos, sendo desprovido de ação analgésica. Quimicamente, é o único agente anestésico venoso que pode ser usado tanto na indução como na manutenção anestésica. $O$ presente trabalho objetivou avaliar freqüencia cardíaca, respiratória, oximetria, pressão arterial média, volume minuto e volume corrente em cães pré-medicados com acepromazina e fentanil e anestesiados por infusão contínua de propofol. Dez cães foram submetidos à medicação pré-anestésica com acepromazina $\left(0,1 \mathrm{mg}^{-\mathrm{kg}^{-1}}\right)$ e fentanil $\left(0,01 \mathrm{mg} \cdot \mathrm{kg}^{-1}\right)$, indução $\left(3,16 \mathrm{mg} \cdot \mathrm{kg}^{-1}\right)$ e manutenção anestésica com propofol em infusão contínua por noventa minutos, na velocidade de $0,4 \mathrm{mg} \cdot \mathrm{kg}^{-1} . \mathrm{min}^{-1}$. Os parâmetros foram mensurados imediatamente após a indução, 10,20,30,60 e 90 minutos após; final da infusão e 30 minutos após o seu término. Os parâmetros foram analisados por análise de variância para valores repetidos e as médias foram analisadas pelo teste de Tuckey em nível de 5\%. O protocolo utilizado não produziu variações estatisticamente significativas em nenhum dos parâmetros analisados. Um animal apresentou apnéia durante a indução. Embasado nesses resultados, verifica-se que o presente protocolo é seguro e eficaz para a realização de anestesia venosa em caninos.
\end{abstract}

Palavras-chave: cães, propofol, infusão contínua, acepromazina, fentanil.

\section{SUMMARY}

Propofol (2,6 diisopropylphenol) is an ultra short duration hypnotic agent that produces sedation and hypnosis similar to barbituric agent, but lacks analgesic action. This is a chemically unique anesthetic agent that can be used for induction and anesthetic maintenance. The objective of this research was to evaluate the cardiac and respiratory rate, oximetry, mean arterial blood pressure and tidal volume and minute volume in dogs premedicated with acepromazine and fentanyl and anesthetized by continuous infusion by propofol. Ten dogs were submitted to anesthesia by continuous propofol infusion during ninety minutes. Using acepromazine $\left(0.1 \mathrm{mg} . \mathrm{kg}^{-1}\right)$ and fentanyl $\left(0.01 \mathrm{mg}^{\mathrm{kg}} \mathrm{kg}^{-1}\right)$ as pre anesthetic drugs, anesthesia was induced using propofol $\left(3.16 \mathrm{mg} . \mathrm{kg}^{-1}\right)$ and maintained by continuous infusion of propofol in a rate of $0.4 m g . \mathrm{kg}^{-1} . \mathrm{min}^{-1}$. The parameters were measured immediately after induction, at 10, 20, 30, 60 and 90 minutes following induction; by the end of infusion and 30 minutes after the end of infusion. The parameters were analyzed by analysis of variance for repeated measures and the means were analyzed by the Tuckey test to 5\% of significance. The protocol used had no statistical effect on the parameters analyzed. One animal showed apnea during the induction phase. The results obtained in this experiment suggest that this protocol might be safely and efficiently used for routine intravenous anesthesia in dogs.

Key words: dogs, propofol, continuous infusion, acepromazine, fentanyl.

\section{INTRODUÇÃO}

Os protocolos de anestesia geral venosa tiveram seu uso reduzido com a produção dos anestésicos inalatórios halogenados, os quais apresentam menores efeitos adversos para os pacientes, como a indução e recuperação mais rápida e tranquiila, o que

\footnotetext{
${ }^{1}$ Médico Veterinário, MS. Professor de Anestesiologia e Terapêutica Veterinária da Universidade de Passo Fundo (UPF). E-mail: jspires@upf.tche.br. Autor para correspondência.

${ }^{2}$ Médico Veterinário, Doutor. Professor Titular, Departamento de Clínica de Pequenos Animais, Uniniversidade Federal de Santa Maria (UFSM), Santa Maria, RS.

${ }^{3}$ Médico Veterinário, MS. Aluno do Programa de Pós-graduação em Medicina Veterinária UFSM.

${ }^{4}$ Médico Veterinário, Aluno do Programa de Pós-graduação em Medicina Veterinária UFSM.
} Recebido para publicação em 23.04.99. Aprovado em 15.03.00. 
permite um controle maior sobre o plano da anestesia. Em alguns casos, como o isofluorano, desfluorano e sevofluorano, a biotransformação pouco interfere na excreção do agente anestésico por ele ser eliminado totalmente pela respiração (STOELTING, 1997).

Recentemente, a anestesia geral venosa vem sendo empregada em maior escala em humanos, oferecendo vantagens como efeitos mais específicos, menor hepatoxidade e, principalmente a ausência de poluição nas salas de cirurgia. Além disso, os membros da equipe cirúrgica expostos aos gases exalados pelos sistemas anestésicos podem apresentar alterações de humor, diabetes mellitus, cirrose hepática e mutações genéticas (HARSFIELD $\boldsymbol{e t}$ al., 1996).

Embora há diversas situações em que a anestesia inalatória na prática cirúrgica veterinária é indicada, há uma clara demanda por um protocolo seguro de anestesia venosa para ser utilizada em caninos saudáveis, em procedimentos cirúrgicos de curta ou média duração (HELLEBREKERS \& SAP, 1997).

O maleato de acepromazina, provavelmente a fenotiazina mais difundida em Medicina Veterinária, vem sendo utilizado em cães, gatos, bovinos e eqüinos, há mais de trinta anos (MEYER, 1997), juntamente com o hidrocloridrato de prometazina, clorpromazina e levomepromazina (BOOTH, 1992). PLUMB (1995) relata que a acepromazina é um agente neuroléptico com mecanismo de ação pouco estudado. As fenotiazinas bloqueiam os receptores pós-sinápticos da dopamina no sistema nervoso central e deprimem as zonas do sistema reticular ativador que controlam a temperatura corporal, metabolismo basal, emese, tono vasomotor, balanço hormonal e sistema de vigília. Mesmo causando esse grau de depressão há relatos de reações idiossincrásicas com acepromazina, (MEYER, 1997). Segundo BOOTH (1992), o maleato de acepromazina é mais potente que a clorpromazina, causando alterações acentuadas na frequiência respiratória dos caninos, mas sem alterações na $\mathrm{PaCO}_{2}$, $\mathrm{pH}, \mathrm{PaO}_{2}$ e na saturação da hemoglobina. As doses recomendadas para cães como medicação préanestésica são $0,03-0,05 \mathrm{mg} \cdot \mathrm{kg}^{-1}$ via intramuscular (IM) ou 1 - $3 \mathrm{mg} \cdot \mathrm{kg}^{-1}$ via oral (VO) (HALL \& CLARKE, 1983); 0,1 - 0,2mg. $\mathrm{kg}^{-1}$ via intravenosa (IV) empregando-se dose total de no máximo $3 \mathrm{mg}$ e 0,055 - 0,11mg kg-1 IV, IM ou subcutâneo (SC) (PLUMB, 1995).

Os agonistas opióides agem em receptores opióides estereoespecíficos presentes nos sítios pré e pós-sinápticos no sistema nervoso central e no sistema nervoso periférico (STOELTING, 1997). O fentanil, é um potente $\mu$ agonista opióide sintético com potência cerca de 100 vezes maior que a morfina, tem início de ação mais rápido devido as suas propriedades farmacocinéticas (BOOTH, 1992; STOELTING, 1997; PLUMB, 1995). O uso de doses múltiplas ou infusões contínuas de fentanil pode causar a saturação dos sítios tissulares inativos, fazendo com que se torne um opióide de longa duração. Tem como vantagem não causar liberação de histamina como a morfina, sendo suas maiores desvantagens a depressão respiratória e rigidez da musculatura esquelética (BOOTH, 1992; STOELTING, 1997; PLUMB, 1995). PLUMB (1995) relata as doses de 0,11 - 0,15mL. $\mathrm{kg}^{-1}$ de fentanil, sempre associado com droperidol, para analgesia e tranqüilização; e para anestesia geral $1 \mathrm{~mL}$ para $18,2 \mathrm{~kg}$, seguido de $12,5 \mathrm{mg} \cdot \mathrm{kg}^{-1}$ de tiopental sódico.

O propofol (2,6 diisopropilfenol), um agente anestésico de ultra curta ação, hipnótico, não barbitúrico e sem semelhança com qualquer outra droga, levemente solúvel em água, é utilizado em caninos (HALL \& CHAMBERS, 1987; WATKINS et al., 1987; GOODCHILD \& SERRAO, 1989; CHAMBERS, 1989; FLECKNELL et al., 1990; ROBERTSON et al., 1992; WATNEY \& PABLO, 1992; BOTELHO et al., 1996).

Quimicamente, o propofol é o único agente anestésico que pode ser usado na indução em forma de bolus como na manutenção anestésica (BRANSON \& GROSS, 1994), na forma de bolus intermitentes (BOTELHO et al., 1996) ou infusão contínua IV (HALL \& CHAMBERS, 1987; WATKINS et al., 1987; GOODCHILD \& SERRAO, 1989; CHAMBERS, 1989; FLECKNELL et al., 1990; ROBERTSON et al., 1992; BRANSON $\&$ GROSS, 1994). Isso se deve às suas características farmacocinéticas que o isentam de efeito cumulativo ao contrário, do tiopental sódico (DUKE, 1995).

O propofol causa rápida perda de consciência, em 20 - 40 segundos, durante a aplicação intravenosa. Após sua administração inicial em bolus, a concentração plasmática declina rapidamente devido à redistribuição a partir do cérebro para outros tecidos altamente perfundidos. A velocidade de eliminação do propofol, para a redistribuição, é similar a do tiopental sódico, sendo que o seu metabolismo é 10 vezes mais rápido (DUKE, 1995).

GOODCHILD \& SERRAO (1989) relatam que a indução da anestesia com propofol foi seguida de diminuição da pressão arterial em associação com o decréscimo do débito cardíaco e da resistência vascular periférica. ROBERTSON $\boldsymbol{e t}$ al. (1992) descrevem que os animais desenvolveram apnéia após a indução, sendo que a pressão arterial 
não sofreu alterações, mas os batimentos cardíacos e os movimentos respiratórios diminuíram significativamente; entretanto, KEEGAN \& GREENE (1993) descrevem que o propofol não causa alterações cardiovasculares e respiratórias em cães anestesiados em infusão contínua.

HALL \& CHAMBERS (1987) utilizaram três grupos com $0,3,0,35,0,4 \mathrm{mg} \cdot \mathrm{kg}^{-1} \cdot \mathrm{mim}^{-1}$ de propofol, respectivamente, WATKINS et al. (1987) usaram $0,806 \mathrm{mg} \cdot \mathrm{kg}^{-1} \cdot \mathrm{mim}^{-1}$ de propofol sem medicação pré-anestésica, GOODCHILD \& SERRAO (1989) utilizaram na sua experimentação as seguintes doses $20,40,80,160 \mathrm{mg} \mathrm{kg}^{-1} \mathrm{~h}^{-1}$, respectivamente.

A literatura cita o uso da infusão de propofol associado a um fármaco como medicação préanestésica .Esse trabalho teve por objetivo avaliar as alterações nos parâmetros de freqüência cardíaca e respiratória, oximetria, pressão arterial média e ventilometria causadas pela infusão contínua de propofol em cães por noventa minutos pré-medicados com acepromazina e fentanil.

\section{MATERIAL E MÉTODOS}

Foram utilizados 10 cães, 6 machos e 4 fêmeas, com peso médio de $9,61 \pm 1,75 \mathrm{~kg}$, adultos, sem raça definida e provenientes do Biotério Central da Universidade Federal de Santa Maria. Os animais foram previamente desverminados e considerados hígidos por exames clínico e laboratorial, sendo mantidos alojados individualmente durante todo o experimento.

Os animais foram submetidos a jejum sólido prévio de 12 horas e a medicação pré-anestésica com cloridrato de acepromazina ${ }^{a}$ e citrato de fentanila ${ }^{\mathrm{b}}$, na dose de $0,1 \mathrm{mg}$ e $0,01 \mathrm{mg} \mathrm{kg}^{-1}$, respectivamente, pela via IM. Quando pesavam mais de $10 \mathrm{~kg}$, foi utilizado um total de $1 \mathrm{mg}$ de acepromazina. Após 20 minutos, um cateter ${ }^{\mathrm{c}} 20 \mathrm{G}$ foi colocado na veia cefálica para a indução e infusão de propofol ${ }^{\mathrm{d}}$. Posteriormente à colocação do cateter, foi induzida a anestesia com propofol na velocidade de $15 \mathrm{mg}$ a cada 10 segundos até atingir o segundo plano do terceiro estágio anestésico. Em seguida, os animais foram colocados em decúbito lateral direito, intubados e conectados a um sistema de Bain e receberam $\mathrm{O}_{2}$ a $100 \%$ com fluxo de $200 \mathrm{~mL} \mathrm{~kg}^{-1} \mathrm{mim}^{-1}$; após iniciou-se a infusão de propofol na velocidade de $0,4 \mathrm{mg} \mathrm{kg}^{-1} \mathrm{~min}^{-1}$, durante 90 minutos, controlado por bomba de infusão ${ }^{\mathrm{e}}$. Ao final desse período, foram avaliados os tempos para extubação e a recuperação até a posição quadrupedal.

Os tempos para o registro dos dados foram T0 (imediatamente após a indução e intubação orotraqueal), T1 (aos 10 minutos), T2 (aos 20 mi- nutos), T3 (aos 30 minutos), T4 (aos 60 minutos), T5 (aos 90 minutos final da infusão) e T6 (30 minutos após o término da infusão). Os parâmetros mensurados nos tempos de avaliação foram as freqüências cardíaca (FC) e respiratória (FR), pressão arterial média (PAM), saturação da hemoglobina pelo oxigênio $\left(\mathrm{Sp} . \mathrm{O}_{2}\right)$, volume minuto $(\mathrm{VM})$ e volume corrente (VC). Os valores referentes a FC foram obtidos através de auscultação e/ou palpação do pulso arterial periférico na artéria femural. Para a obtenção dos valores de FR, observaram-se os movimentos respiratórios torácicos por minuto.

Para verificação da PAM invasiva, os caninos tiveram cateterizada a artéria femural com cateter $20 \mathrm{G}$ previamente perfundido com uma solução contendo 4 UI de heparina ${ }^{\mathrm{f}} \mathrm{mL}^{-1}$; o cateter foi conectado a um equipo, com uma cânula de três vias, e a um manômetro aneróide ${ }^{\mathrm{g}}$ onde os valores foram medidos em $\mathrm{mmHg}$.

O VM foi obtido, através de um ventilômetro $^{\mathrm{h}}$ conectado ao traqueotubo ou a uma máscara facial, e o VC foi realizado dividindo-se o VM pela FR e o produto pelo peso do animal. A Sat. $\mathrm{O}_{2}$ da hemoglobina foi mensurada de forma não invasiva com Oxímetro de pulso ${ }^{i}$, sendo o sensor colocado na língua do animal, durante a anestesia, e no lábio superior para a última mensuração.

Os parâmetros foram analisados por análise de variância para valores repetidos e as médias analisadas pelo teste de Tuckey em nível de 5\% de significância.

\section{RESULTADOS E DISCUSSÃO}

A combinação do cloridrato de acepromazina e citrato de fentanila, na medicação préanestésica, produziu adequada sedação, evitando que os animais apresentassem reações ao manuseio, à colocação dos cateteres e reduzindo a dose necessária de propofol para indução à anestesia. No presente experimento, a dose foi reduzida para 3,16 \pm $0,81 \mathrm{mg} \cdot \mathrm{kg}^{-1}$ em média contra $6 \mathrm{mg} \cdot \mathrm{kg}^{-1}$ citados por PLUMB (1995), 5,95mg. $\mathrm{kg}^{-1}$ relatados por WATKINS et al. (1987), 4,7 + 1,3mg. $\mathrm{kg}^{-1}$ utilizados por WATNEY \& PABLO (1992) e $8 \mathrm{mg}^{-\mathrm{kg}^{-1}}$ usados por QUANDT et al. (1998), que não utilizam medicação pré-anestésica. Os resultados obtidos apoiamse nos de HELLEBREKERS \& SAP (1997) que usaram a medetomidina como medicação préanestésica, com três diferentes agentes indutores, sendo que a dose de propofol necessária à indução, foi de $2,0 \mathrm{mg} \cdot \mathrm{kg}^{-1}$. Obteve-se redução de $47 \%$ na dose de propofol, com o presente protocolo, redução maior do que apenas com o uso da acepromazina realizado por WATKINS et al. (1987). No entanto, 
LUNA et al. (1998) utilizaram $5 \mathrm{mg} \cdot \mathrm{kg}^{-1}$, quando associado à xilazina, podendo ser conseqüência da velocidade com que foi administrado o propofol para a indução anestésica conforme DUKE (1995).

A verificação do plano anestésico foi realizada através da relaxação dos músculos da mandíbula e pela redução da intensidade dos reflexos palpebrais e corneal. Quando ocorria a redução da atividade reflexógena e relaxação da mandíbula, era realizada a intubação orotraqueal. Não foi necessária dosagem suplementar de propofol durante o período da infusão.

A taxa de infusão utilizada foi constante em $0,4 \mathrm{mg} \cdot \mathrm{kg}^{-1} \cdot \mathrm{min}^{-1} \quad$ sem estimulação cirúrgica, taxa igual a relatada por HALL \& CHAMBERS (1987), que fizeram uso de três taxas de infusão 0,3 , $0,35, \quad 0,4 \mathrm{mg} \cdot \mathrm{kg}^{-1} \cdot \mathrm{min}^{-1}$, respectivamente, e observaram que somente a taxa de $0,4 \mathrm{mg} . \mathrm{kg}^{-}$ ${ }^{1} . \mathrm{min}^{-1}$ produziu anestesia cirúrgica em cães. AGUIAR et al. (1993) utilizaram três taxas de infusão $0,2,0,3$ e $0,4 \mathrm{mg} \cdot \mathrm{kg}^{-1} \cdot \mathrm{min}^{-1}$, sendo que as duas taxas inferiores podem ser utilizadas com o emprego de fármaco analgésico como citado por FLECKNELL et al. (1990), que usaram propofol e alfentanil em infusão contínua, reduzindo a velocidade de infusão do propofol para 0,14 a $0,18 \mathrm{mg} \cdot \mathrm{kg}^{-1} \cdot \mathrm{min}^{-1}$ e 0,002 a $0,003 \mathrm{mg} \cdot \mathrm{kg}^{-1} \cdot \mathrm{min}^{-1}$, respectivamente. KEEGAN \& GREENE (1993) usaram $0,44 \mathrm{mg} \cdot \mathrm{kg}^{-1} \cdot \mathrm{min}^{-1}$ de velocidade de propofol durante duas horas, taxa superior à utilizada na presente experimentação.

O parâmetro freqüência cardíaca (Tabela 1) apresentou-se no tempo T0 elevado. Seguindo-se de uma pequena redução no tempo T1 e mantendose constante até o tempo T5 em relação ao T0, sendo que no tempo T5 foi a menor freqüência apresentada, retornando, no tempo T6, ao valor inicial; entretanto, em nenhum dos tempos estudados ocorreram diferenças estatísticas significantes.

A freqüência cardíaca apresentou pequena redução nos tempos estudados, não sendo diagnosticadas alterações importantes. Resultados semelhantes aos de ROBERTSON et al. (1992) os quais verificaram que os animais da raça Greyhounds apresentaram bradicardia com diferença significativa entre os valores dos batimentos cardíacos nos tempos estudados; isso, provavelmente, deve-se aos animais dessa raça apresentarem um tono vagal alto, onde ocorre ação do propofol e os animais de outras raças não apresentaram nenhuma diferença nos valores. É relatada por KEEGAN \& GREENE (1993) a redução da freqüência cardíaca, com o passar dos tempos estudados, caracterizando o efeito inotrópico abela 1 - Variações dos valores médios e desvios-padrões dos parâmetros frequiência cardíaca (FC), pressão arterial média (PAM), frequência respiratória (FR), oximetria $\left(\mathrm{Sp} . \mathrm{O}_{2}\right)$, volume minuto $(\mathrm{VM})$ e volume corrente (VC) durante os tempos estudados em cães pré-medicados com acepromazina e fentanil e anestesiados por infusão contínua de propofol.

\section{TEMPOS}

$\begin{array}{llllllll}\text { Parâmetros } & \text { T0 } & \text { T1 } & \text { T2 } & \text { T3 } & \text { T4 } & \text { T5 } & \text { T6 }\end{array}$

$\begin{array}{cccccccc}\text { FC }(\mathrm{bpm}) & 106,8 & 97,1 & 97,9 & 97,2 & 99,9 & 88,9 & 102,5 \\ & \pm 28,1 & \pm 26,4 & \pm 25,7 & \pm 23,9 & \pm 23,2 & \pm 30,4 & \pm 11,4 \\ \text { PAM (mmHg) } & 88,5 & 84,3 & 85,5 & 84,4 & 81,8 & 74,5 & ---- \\ & \pm 15,1 & \pm 8,87 & \pm 8,87 & \pm 6,99 & \pm 8,76 & \pm 8,07 & --- \\ \text { FR.(mpm) } & 16,2 & 19,3 & 22,8 & 22,4 & 20,8 & 21,7 & 20,1 \\ & \pm 11,43 & \pm 7,98 & \pm 17,90 & \pm 17,09 & \pm 20,09 & \pm 21,09 & \pm 6,85 \\ \text { Sp.O } 2(\%) & 93,3 & 94,5 & 94,7 & 93,8 & 94,2 & 95,2 & 95,1 \\ & \pm 2,26 & \pm 1,87 & \pm 2,21 & \pm 2,74 & \pm 1,87 & \pm 1,75 & \pm 2,18 \\ \text { VM }\left(1 . \mathrm{min}^{-1}\right) & 1,78 & 1,82 & 1 ; 94 & 1,82 & 1,87 & 1,85 & 2,01 \\ & \pm 0,43 & \pm 0,51 & \pm 0,77 & \pm 0,73 & \pm 0,71 & \pm 0,64 & \pm 0,58 \\ \text { VC }\left(\mathrm{mlkg}^{-1} \mathrm{~min}^{-1}\right) & 11,76 & 10,50 & 10,88 & 10,04 & 12,18 & 11,37 & 11,39 \\ & \pm 2,78 & \pm 2,48 & \pm 3,16 & \pm 2,12 & \pm 3,96 & \pm 3,87 & \pm 4,33\end{array}$

Valores expressos em médias e desvios-padrões.

- imediatamente após a indução e intubação orotraqueal, T1 - 10 minutos, T2 minutos, T3 - 30 minutos, T4 - 60 minutos, T5 - 90 minutos final da infusão 6 - 30 minutos após o término da infusão.

negativo que o propofol apresenta. Entretanto, utilizando $0,4 \mathrm{mg} \cdot \mathrm{kg}^{-1} \cdot \mathrm{min}^{-1}$, HALL \& CHAMBERS (1987) relataram a possibilidade de ocorrência de estimulação simpática durante a infusão de propofol, devido aos valores médios dos batimentos cardíacos se elevarem nos tempos em que foram mensurados. AGUIAR et al. (1993) também relataram o aumento da freqüência cardíaca especialmente após os 20 minutos de anestesia, coincidindo com a diminuição dos valores da pressão arterial.

GOODCHILD \& SERRAO (1989), utilizando altas doses de infusão de propofol, comprovaram que as alterações cardiovasculares são decorrentes da ação direta da droga, que atua em todos os nervos do sistema nervoso autônomo venomotor e sobre o controle do tono arterial.

A pressão arterial média (PAM) (Tabela 1) manteve-se constante durante os tempos $\mathrm{T} 0$ a $\mathrm{T} 4$, diminuindo no tempo T5, sem variação estatística significante. O tempo T6 não foi mensurado devido à impossibilidade se manter a artéria femural canulada durante o período de recuperação. Esses resultados são similares aos relatados por (FLECKNELL et al., (1990), ROBERTSON et al., (1992), KEEGAN \& GREENE, (1993) e QUANDT $\boldsymbol{e}$ t al., (1998) que também relatam que a PAM não apresentou alterações estatísticas significativas durante o período da anestesia; entretanto LUNA et al. (1998) reportam a ocorrência de uma discreta redução no valor da PAM aos 20 minutos de infusão da combi- 
nação de propofol e quetamina. Indicando a segurança desse fármaco em relação à pressão arterial.

Os animais apresentaram uma elevação nos valores médios dos movimentos respiratórios (Tabela 1) nos tempos T1 a T6 em relação a T0, porém não apresentaram diferenças significantes entre os tempos estudados. Dois animais apresentaram taquipnéia, um nos tempos T0 (40mpm), T2 (52mpm) e T3 (48mpm) e outro entre os tempos T1 (40mpm), T2 (60mpm), T3 (60mpm), T4 (75mpm) e T5 (80mpm), retornando ao normal no tempo T6. Um animal apresentou apnéia no tempo T0. Outro animal apresentou apnéia no tempo T5, durando 70 segundos, sendo necessário instituir-se ventilação assistida até a volta dos movimentos respiratórios. A ocorrência de apnéia durante a indução à anestesia com propofol é relatada por HALL \& CHAMBERS (1987); FLECKNELL et al. (1990); AGUIAR et al. (1993); KEEGAN \& GREENE (1993); BOTELHO et al. (1996); MUIR III \& GADAWSKI (1998) e QUANDT et al. (1998) demonstram o elevado grau de depressão que o propofol causa no centro respiratório. $\mathrm{Na}$ presente experimentação, somente um animal apresentou apnéia na indução, com duração de aproximadamente três minutos. A redução dos casos de apnéia provavelmente se deva à velocidade de administração do propofol durante a indução, que era de $15 \mathrm{mg}(1.5 \mathrm{~mL})$ a cada 10 segundos. MUIR III \& GADAWSKI (1998), utilizando 20mg a cada 10 segundos, observaram um maior número de episódios de apnéia durante a indução anestésica, indicando que a velocidade de administração do propofol na indução pode estar relacionada com a depressão dos centros respiratórios.

Os parâmetros volume minuto e volume corrente (Tabela 1) não apresentaram diferenças significantes entre os tempos estudados. Os valores do volume corrente apresentaram-se constantes nos tempos estudados. Nos animais que apresentaram episódios de taquipnéia, ocorreu diminuição do volume corrente mesmo com a elevação do parâmetro volume minuto. Valores médios de volume corrente desse experimento encontram-se dentro dos valores considerados normais, por MUIR III \& HUBBEL (1995) que variam entre 10 a $15 \mathrm{~mL} \cdot \mathrm{kg}^{-1}$ $\min ^{-1}$, enquanto que SHORT (1987) relata a variação entre 10 a $20 \mathrm{~mL} \cdot \mathrm{kg}^{-1} \cdot \mathrm{min}^{-1}$.

Os valores do volume minuto elevaram-se em T1 em relação a T0, permanecendo constantes até T5, voltando a subir em T6 em relação a T5. Os valores de volume minuto foram obtidos através de um ventilômetro de Wright, que, segundo HALL \& CLARKE (1983) e SHORT (1987), é o instrumento adequado para tal finalidade. Essas variações, embora não sejam estatisticamente significantes, demostram que o propofol atua consideravelmente sobre o centro respiratório.
A saturação periférica de $\mathrm{O}_{2}$ da hemoglobina permaneceu constante durante todo o experimento (Tabela 1), não apresentando diferenças estatísticas significantes entre os tempos estudados, permanecendo dentro dos valores considerados fisiológicos, que, segundo PLUMB (1995) e GUYTON \& HALL (1997), oscila entre 92 a 99\%, entretanto LUMB \& JONES (1996) referem um valor não inferior a $95 \%$. O fato da oximetria não apresentar variações, provavelmente esteja relacionado ao uso do oxigênio e à perfusão tecidual adequada, como demonstram os valores da PAM durante o período anestésico. Resultado semelhante foi encontrado por AGUIAR et al. (1993) em que todos os animais permaneceram com os valores de oximetria dentro dos valores normais. Somente um animal apresentou um valor de saturação abaixo de $90 \%$ devido à parada respiratória mais prolongada, quando foi instituída a ventilação controlada, retornando os valores aos normais. CHAMBERS (1989), utilizando alfentanil e propofol para obter a indução anestésica, observou apnéia em três animais durante três minutos, resultando também em valores reduzidos de oximetria.

Os animais começaram a apresentar sinais de reflexos laríngeos, em média, com $9 \pm 3,04$ minutos após o final da infusão, quando foi realizada a extubação. O tempo decorrido do término da infusão até o momento da extubação foi de nove minutos, que é superior aos obtidos por AGUIAR et al. (1993) os quais relataram uma variação de cinco a sete minutos; esse tempo demonstra que o propofol apresenta pouco efeito cumulativo. Em um estudo comparativo, QUANDT et al. (1998) relatam que os animais anestesiados com propofol apresentam tempo de recuperação menores que os anestesiados com tiopental sódico.

Os animais demoraram em média 30, 4 + 3,45 minutos após o final da infusão para retornarem à estação; que foi considerado a partir do término da infusão do propofol. Os tempos encontrados foram inferiores aos relatados por AGUIAR et al. (1993) $70,4+14,4$ minutos utilizando a mesma velocidade de infusão. O tempo menor na recuperação, encontrado neste experimento, pode estar relacionado com a medicação pré-anestésica utilizada.

\section{CONCLUSÃO}

Nas condições em que foi realizado este experimento, foi possível concluir que a associação de acepromazina, fentanil e propofol é segura para utilização em caninos por não promover alterações na freqüência cardíaca, pressão arterial média e saturação periférica da hemoglobina pelo oxigênio desde que seja realizada com equipamentos precisos, assegurando precisão na velocidade de infusão. É necessário intubar os animais devido à depressão respiratória causada pelo propofol. 


\section{AGRADECIMENTOS}

Ao laboratório ICI-Wellcome Zeneca que gentilmente sedeu as amostras de propofol para a realização desse experimento.

\section{FONTES DE AQUISIÇÃO}

a Acepran - Univet S/A - Indústria Veterinária. São Paulo, SP. b Fentanil - Cristália - Produtos Químicos e Farmacêuticos Ltda. Itapira, SP

c Introcan - Laboratórios B. Braum S.A - São Gonçalo, RJ. d Diprivan - Laboratórios Wellcome - ICI Ltda. Cotia, SP.

e Farms 600- Lifemed. Pesquisas Médicas Ind. e Comércio Ltda. São Paulo, SP

f Heparin- Cristália - Produtos Químicos e Farmacêuticos Ltda. Itapira, SP.

g Manômetro Aneróide - Van Ross Ltda. - São Paulo, SP.

h Wrigth's Ventilometer - British Oxigen Company, London UK.

i Nellcor N 200 - Nellcor Inc. Hayward, California. USA.

\section{REFERÊNCIAS BIBLIOGRÁFICAS}

AGUIAR, A.J.A., LUNA, S.P.L., OLIVA, V.N.L.S. $\boldsymbol{e} \boldsymbol{t} \boldsymbol{a l}$. Anestesia por infusão contínua com propofol em cães após medicação pré-anestésica com levomepromazina. Journal of Veterinary Anaesthesiology, v.20, p.26-28, 1993.

BOOTH, N.H. Agentes psicotrópicos. In: BOOTH, N.H. McDONALD, L. E. Farmacologia e terapêutica em veterinária. 6 ed. Rio de Janeiro : Guanabara Koogan, 1992. Cap.17. p.289-314.

BOTELHO, R.P. NASCIMENTO, M.D., MARSICO $\mathrm{F}^{\circ}$, F. Propofol: avaliação clínica e laboratorial em cães. Revista Brasileira Ciências Veterinária, v.3, n.3, p.81-87, 1996.

BRANSON, K.R., GROSS, M.E. Propofol in veterinary medicine. Journal of the American Veterinary Medical Association, v.204, n.12, p.1888-1890, 1994.

CHAMBERS, J.P. Induction of anaesthesia in dogs with alfentanil and propofol. Journal of the Association of Veterinary Anaesthetists of Great Britain and Ireland. v.16, p.14-17, 1989.

DUKE, T. A new intravenous anesthetic agent: Propofol. Canine Veterinary Journal. v.36, p.181-183, 1995.

FLECKNELL, P.A., KIRK, A.J.B., FOX, C.E. et al. Long-term anaesthesia with propofol and alfentanil in the dog and its partial reversal with nalbuphine. Journal of the Association of Veterinary Anaesthetists of Great Britain and Ireland. v.17, p.11-16, 1990.

GUYTON, A.C., HALL, J.E. Tratado de fisiologia médica. 9 ed. Rio de Janeiro : Interamericana, 1997. Cap.40: transporte de oxigênio e de dióxido de carbono no sangue e em outros líquidos corporais: p.465-474.

GOODCHILD, C.S., SERRAO, J.M. Cardiovascular effects of propofol in the anaesthetized dog. British Journal of Anaesthesia. v.63, p.87-92, 1989.

HALL, L.W., CLARKE, K.W. Veterinary anaesthesia. 3.ed. London : Bailliére Tindal, 1983. 422p.
HALL, L.W., CHAMBERS, J.P. A clinical trial of propofol infusion anaesthesia in dogs. Journal of Small Animal Practice, v.28, p.623637, 1987.

HARSFIELD, S., McGRATH, C. GAYNOR, J. et al. Commentary and recommendations on control of waste anesthetic gases in the workplace. Journal of the American Veterinary Medical Association. v.209, n.1, p.75-77, 1996.

HELLEBREKERS, L.J., SAP, R. Medetomidine as a premedicant for ketamine, propofol or fentanyl anaesthesia in dogs. Veterinary Record, v.24, p.545-548, 1997

KEEGAN, R.D., GREENE, S.A. Cardiovascular effects of a continuous two hour propofol infusion in dogs comparison with isoflurane anaesthesia. Veterinary Surgery, v.22, n.6, p.537-543, 1993.

LUMB, W.V. JONES, E.W. Veterinary anesthesia. 3 ed. Baltimore : Williams \& Wilkins, 1996. 928p.

LUNA, S.L.P., GASPARINI, S.S., MASSONE, F. et al. Anestesia intravenosa utilizando propofol ou propofol/quetamina em cadelas submetidas à ovariosalpingohisterectomia. In: CONGRESSO BRASILEIRO DO COLÉGIO BRASILEIRO DE CIRURGIA E ANESTESIOLOGIA VETERINÁRIA, 1998. Belo Horizonte, MG. Anai. Belo Horizonte : CBCAV, 1998. 162p. p.8

MEYER, E.K. Rare, idiosyncratic reation to acepromazine in dogs. Journal of the American Veterinary Medical Association. v.210, n.8, p.1114-1115, 1997.

MUIR III, W.W., HUBBEL, J.A.E. Handbook of veterinary anesthesia. 2 ed. Sant Louis : Mosby, 1995. 490p.

MUIR III, W.W., GADAWSKI, J.E. Respiratory depression and apnea induced by propofol in dogs. American Journal of Veterinary Research. v.59, n.2, p.157-161, 1998.

PLUMB, D.P. Veterinary drug handbook. 2 ed. Iowa : Iowa State University, 1995. 790p.

QUANDT, J.E., ROBINSON, E.P., RIVERS, W.J. et al. Cardiorespiratory and anesthetic effects of propofol and thiopental in dogs. American Journal of Veterinary Research, v.59, n.9, p.1137-1143, 1998.

ROBERTSON, S.A., JOHNSTON, S., BEEMSTERBOER, J. Cardiopulmonary, anesthetic, and postanesthetic effects of intravenous of propofol in Greyhounds and non-Greyhounds. American Journal of Veterinary Research, v.53, n.6, p.1027-1032, 1992 .

SHORT, C.E. Principles \& practice of veterinary anesthesia. Baltimore : Williams \& Wilkins, 1987. 669p.

STOELTING, R.K. Manual de farmacologia \& fisiologia na prática anestésica. Porto Alegre : Artes Médicas, 1997. 500 p.

WATKINS, S.B., HALL, L.W., CLARKE, K.W. Propofol as na intravenous anaesthetic agent in dogs. Veterinary Record. v.120, p.326-329, 1987.

WATNEY G.C.G., PABLO, L.S., Median effective dosage of propofol for induction of anesthesia in dogs. American Journal of Veterinary Research, v.53, n.12, p.2320-2323, 1992. 